\title{
Purification of a bacteriocin-like inhibitory substance derived from pediococcus acidilactici Kp10 by an aqueous micellar two-phase system
}

\begin{abstract}
A polymer-salt aqueous two-phase system (ATPS) consisting of polyethylene-glycol (PEG) with sodium citrate was developed for direct recovery of a bacteriocin-like inhibitory substance (BLIS) from a culture of Pediococcus acidilactici Kp10. The influences of phase composition, tie-line length (TLL), volume ratio (VR), crude sample loading, $\mathrm{pH}$ and sodium chloride $(\mathrm{NaCl})$ on the partition behaviour of BLIS was investigated. Under optimum conditions of ATPS, the purification of BLIS was achieved at $26.5 \%$ PEG (8000)/11\% sodium citrate with a TLL of $46.38 \%(\mathrm{w} / \mathrm{w})$, VR of 1.8 , and $1.8 \%$ crude load at $\mathrm{pH} 7$ without the presence of $\mathrm{NaCl}$. BLIS from P. acidilactici Kp10 was successfully purified by the ATPS up to 8.43-fold with a yield of 81.18\%. Given that the operation of ATPS is simple, environmentally friendly and costeffective, as it requires only salts and PEG, it may have potential for industrial applications in the recovery of BLIS from fermentation broth.
\end{abstract}

Keyword: Pediococcus acidilactici; Bacteriocin-like inhibitory substance; Aqueous twophase system; Recovery 